\title{
Acceleration based black-box accident detection and warning system
}

\author{
Kadri Ibrahim, Kadri Boufeldja, Beladgham Mohammed, Dahmane Oussama \\ Data Processing and Telecommunication Laboratory (LTIT), Faculty of Technology, \\ University Tahri Mohammed Bechar, Algeria
}

\section{Article Info \\ Article history: \\ Received Jan 16, 2021 \\ Revised Jul 27, 2021 \\ Accepted Aug 15, 2021 \\ Keywords: \\ Accident warning \\ GPS \\ GSM \\ Image processing \\ Minimum set of data \\ OBD-II standard}

\begin{abstract}
The purpose of this research paper is to develop a black-box accident warning system that utilizes both global system for mobile communication (GSM) and global positioning system (GPS) technologies to identify and deliver a minimum set of data that includes critical data about the accident gathered via various sensors such as the type of accidents, geographic coordinates, time, and velocities (civil protection, hospitals, and police). Additionally, the system makes use of data collected via the OBD-II standard to provide a reliable method for detecting accidents based on vehicle acceleration. Clearly, this system appeared to be the best option for countries with significantly older average vehicle ages. The system was installed in a real vehicle in order to evaluate the collision detection algorithm through the use of abrupt medium-speed braking. The system also includes an image processing system for age and gender prediction based on the Rapsberry Pi and intel neural compute stick 2 (NCS2) that will be published in future work.
\end{abstract}

This is an open access article under the CC BY-SA license.

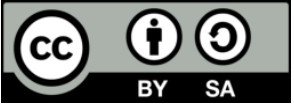

\section{Corresponding Author:}

Kadri Ibrahim

Data Processing and Telecommunication Laboratory (LTIT)

University Tahri Mohammed Bechar, Algeria

Email: kadri.ibrahim@univ-bechar.dz

\section{INTRODUCTION}

Transportation has grown exponentially in recent years in today's society. Regrettably, this was a significant factor in the increase in deadly road collisions [1], [2]. Nearly 1.2 million people are killed in automobile accidents each year. Additionally, between 20 and 50 million individuals are permanently handicapped or seriously harmed [3]. Prior research has established a substantial correlation between traffic accident deaths and the reaction time of intervention services: the time required for intervention units to reach on the scene of the accident [3]. Automated vehicle accident detection and warning systems, often called as eCall systems, can help intervention responders respond more quickly. As a consequence, several lives may be saved and the severity of injuries may be minimized [4], [5].

To facilitate fast action, we introduced an accident detection and warning system (ADWS) capable of transmitting a minimum set of data (MSD) from the vehicle to intervention services [6]. The following data is included in this MSD:

a) Accident type

b) Geographic position (Longitude and latitude)

c) Time of the accident

d) Speed

e) Acceleration/Deceleration 
The proposed method makes use of GPS technology to compute the vehicle's precise longitude and latitude coordinates and transmits them to intervention services through the global system for mobile communication (GSM) network. Additionally, it utilizes the Raspberry Pi to handle acceleration and a number of other data collected through different kinds of sensors [7].

\section{RELATED WORKS}

Emergency call is a call that enables the driver of the vehicle to send a message to the public safety answering point (PSAP) manually or automatically in the case of a significant collision [8].

\subsection{Built-in units}

Automobiles manufacturers are now pursuing integrated (built-in unit) emergency call systems for their latest automobiles. The primary benefit of this kind is the ability to check the status of any sensor installed in the vehicle, including accelerometers and airbag deployment monitors. to establish how an accident occurred.

\subsection{Black-box systems}

Unfortunately, the majority of cars in nations with a much greater average age do not have automated system for detecting accidents and reporting [9]. The optimum option in this situation is to use the 'black-box' eCall solutions, independent or deployable devices that have the specific personal sensor hardware, positioning and communication capabilities [4].

\subsection{Mobile phone-based systems}

Almost every passenger in a vehicle has a smartphone with the increasing use of mobile communications devices. This includes the key technical parts needed by an emergency call system (GPS, accelerometer, GSM communication) [4]. In relation to built-in unit are the benefits and limitations of smartphone accident warning apps, e.g. do not automatically link to in-vehicle sensors, and offer significant information for accident investigations including videos and images. They are not as accurate as other systems, however, since they may generate misleading positive effects [9].

Nemerous researchers are nowadays developing eCall systems. First of all, the mobile phone's applications are the most widespread solutions due to their low cost and ease of use. However, they're the least effective comparing to built-in and black-box solutions. In fact, almost all mobile phone applications employed the integrated smartphone accelerometer's data to perform the accidents detection [9]. Some of them used special machine learning algorithms so as to analyze this data and determine the accident severity [10]-[12]. Others utilized both integrated smartphones and vehicle sensors in the accident detection in order to raise the confidence rate [13]. Therefore, ignoring the use of the in-vehicle sensors in the accident detection procedure causes a big limitation in such systems performances [14].

Secondly, black-box solutions are more complicated in terms of conception and implementation. Whereas, they have more efficiency in terms of the providing accident data quality. They either used specific types of sensors or the in-vehicle sensors, microcontrollers are mainly employed in the conception of this kind of systems to manipulate the different electronic compenents, detect, locate the vehicle accident and send the warning message due to the GPS and GSM networks [15], [16]. Furthermore, both mobile phone and black-box solutions have a major insufficiency as long as they are not using the in-vehicle sensors data in the accident detection phase.

\section{PROPOSED SYSTEM}

Black-box approach presented in this study can significantly reduce reaction time by automatically sending an alarm message indicating the vehicle's location through GPS. This message will be forwarded to intervention services, who will determine the appropriate course of action in response to the warning. Because time is critical in these types of circumstances, this strategy will save significant time. The proposed system design is depicted in Figure 1. In the case of a traffic collision, an algorithm for detecting accidents will process multiple data sources and instruct the Raspberry Pi to generate and deliver a warning message to intervention services via the GSM module carrying the vehicle's accident information. 


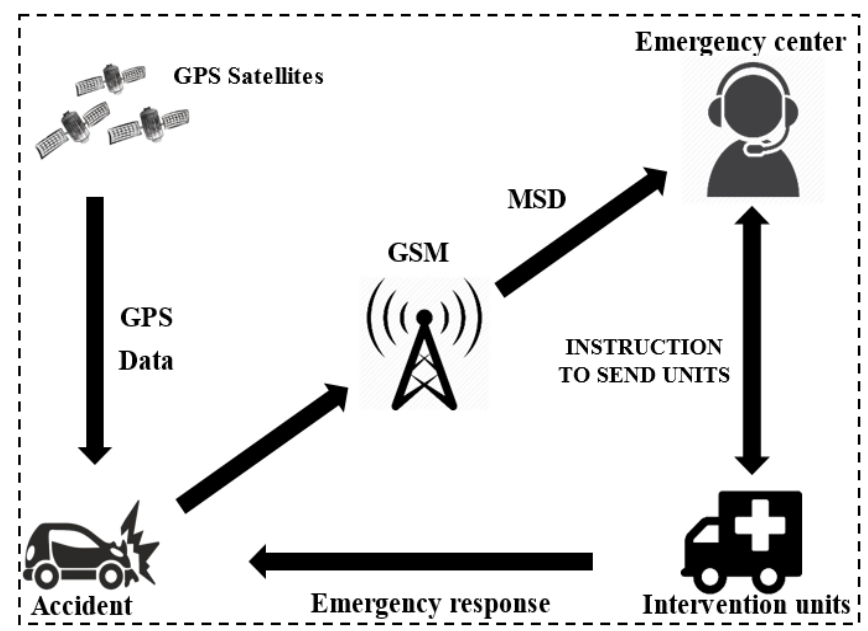

Figure 1. Diagram of the proposed system

\subsection{Hardware architecture}

The black box technology shown in this article can decrease the reaction time by creating an automatic warning message that identifies the vehicle geographic position using a GPS device. This notification will be forwarded to intervention services, who will determine the appropriate course of action to take in response to the alert. Due of the critical nature of time in these types of treatments, this strategy saves considerable time. The hardware architeture of the proposed black box system is shown in Figure 2 . The system is intended as a transportable unit, allowing for easy relocation and maintenance.

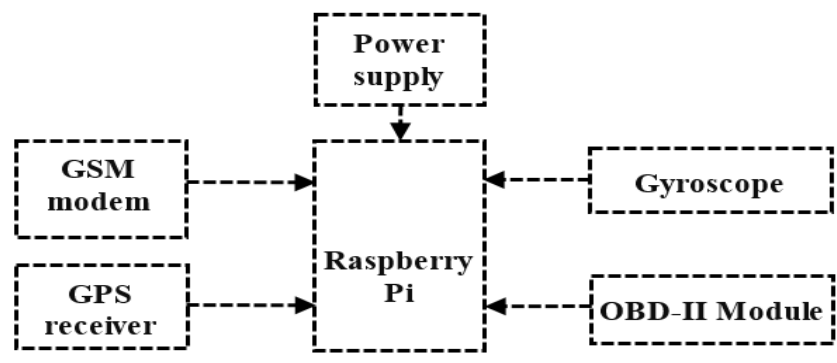

Figure 2. Proposed hardware architecture

\section{GPS FOR ACCIDENT DETECTION SYSTEM}

The global positioning system is a navigational satellite network system established and operated for military and civil uses by the United States Department of Defense [17]. The GPS device indicates the geographic location of the crash by comparing the moment when a number of satellites received signals and triangulating on the already known satellites' locations. The place of the accident may be determined accurately with GPS technology [18]. In line with wuth NMEA specifications, the GPS receptor sends data via universal asynchronous receiver transmitter (UART) and the NMEA defines communication using a variety of distinct expressions [19].

The \$GPRMC phrase of the NMEA 0183 communications protocol is often used for data transmission on GPS devices. The time, place and speed of the \$GPRMC phrase can be removed. Figure 3 shows the \$GPRMC sentence structure [20], [21].

Before using the data, it is necessary to convert it from the format supplied by the \$GPRMC expression (degrees, minutes, and seconds: ddmm.ssss) to decimal coordinates. The longitude and latitude must be converted from the format supplied by the \$GPRMC sentence (degrees, minutes, and seconds: ddmm.ssss) to decimal coordinates (dd.mmssss). 


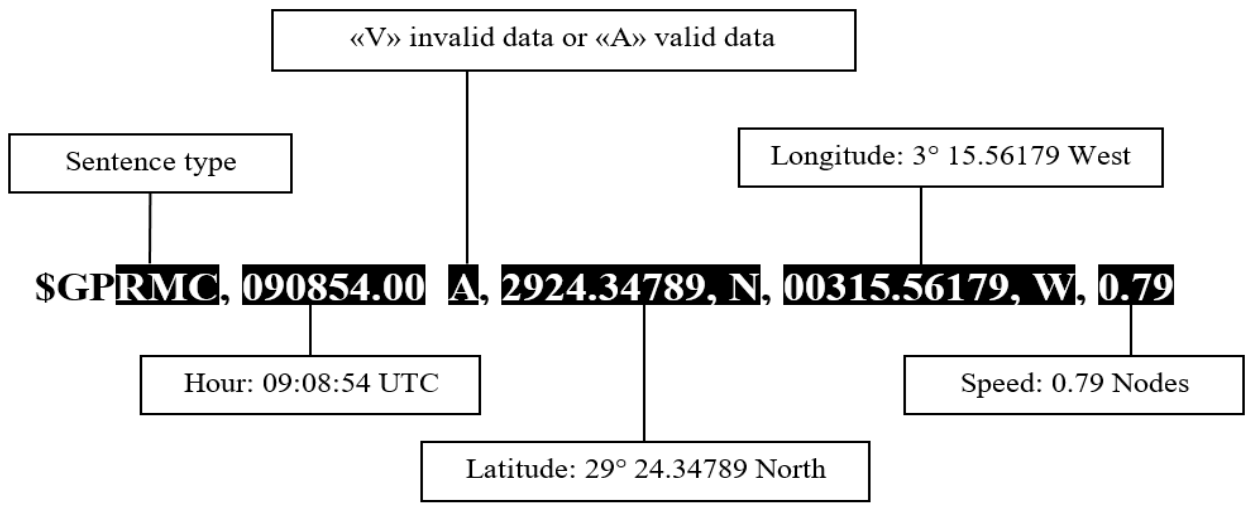

Figure 3. The \$GPRMC sentence

\section{ACCIDENT DETECTION PROCEDURE}

Excessive speed is one of the principal causes of traffic accidents, the distance between the start of braking until the complete standing of the vehicle increases proportionally by speeding up. According to the law of conservation of energy, a moving body has kinetic energy (1). When an accident happens, kinetic energy is transformed to destructive forces, resulting in damage to both the passengers and the vehicle [22].

$$
\text { KineticEnergy }=\frac{1}{2} m v^{2}
$$

The acceleration information can be extracted from three different sources: Accelerometer, GPS data and the in-vehicle sensors [23]. Since the accelerometer and the GPS data are not accurate enough to allow distinguishing between real accidents and other types of events, the perfect solution is to use the speed data obtained from the in-vehicle sensors, and calculate acceleration based on that data. Generally, accident occurs when the detected deceleration is bigger than $5 \mathrm{Gs}$. If the force is above $20 \mathrm{Gs}$, the accident is considered serious. Fatal cases take place in impact above 50 Gs [24].

The proposed procedure to detect the occurrence of vehicle crashes in our system is based on the use of the OBD-II standard. The Raspberry Pi obtains speed information by connecting to the car's OBD-II through the bluetooth connection, acceleration/deceleration was estimated from the speed data acquired every 140 milliseconds using (2) [25], [26].

$$
d_{t 2}=\frac{v_{1}-v_{2}}{t_{1}-t_{2}}
$$

where, $d_{t 2}$ is deceleration at time $t_{2}$ and $v_{1}$ and $v_{2}$ are the speeds at $t_{1}$ time and $t_{2}$ respectively [27].

\section{DATA TRANSMISSION PROCEDURE}

When an accident occurs, the accident warning system must broadcast the accident data to the intervention services in real time in order to provide fast intervention. Based on the estimated acceleration value, a short message service (SMS) is delivered using a GSM modem that provides the longitude and latitude of the incident as well as the time of occurrence, speed, accident type, and severity of the accident.

The Raspberry Pi can communicate with the GSM modem by sending AT commands to it. The 'ATE0' command is used to turn off the echo for the first time. In order to enable text mode (SMS), the command ' $\mathrm{AT}+\mathrm{CMGF}=1$ ' must be entered, and the command ' $\mathrm{AT}+\mathrm{CMGS}$ ' must be entered in order to sends the SMS to a recipient number that has been pre-selected.

\section{DATA TRANSMISSION PROCEDURE}

In this paper, the vehicle acceleration is used as a main factor to predict whether or not accident occurs. In sever accident, the speed decreases from a high value to zero in a very short time lapse [28]. The 
Raspberry Pi monitors the acceleration data obtained through the OBD-II protocol in real-time. The system considers the instance acceleration/deceleration as an accident when it exceeds $\pm 5 \mathrm{~g}$.

The system comprises both gyroscopes and flame sensors in the case of automobile overturn or fire. As a result, the geographic location phase will be triggered, which allows GPS technology to capture the exact location and time of the accident, which will subsequently be transmitted to emergency services via GSM connection. Figure 4 displays a flow diagram of the algorithm for the accident detection and warning system that has been proposed by the author.

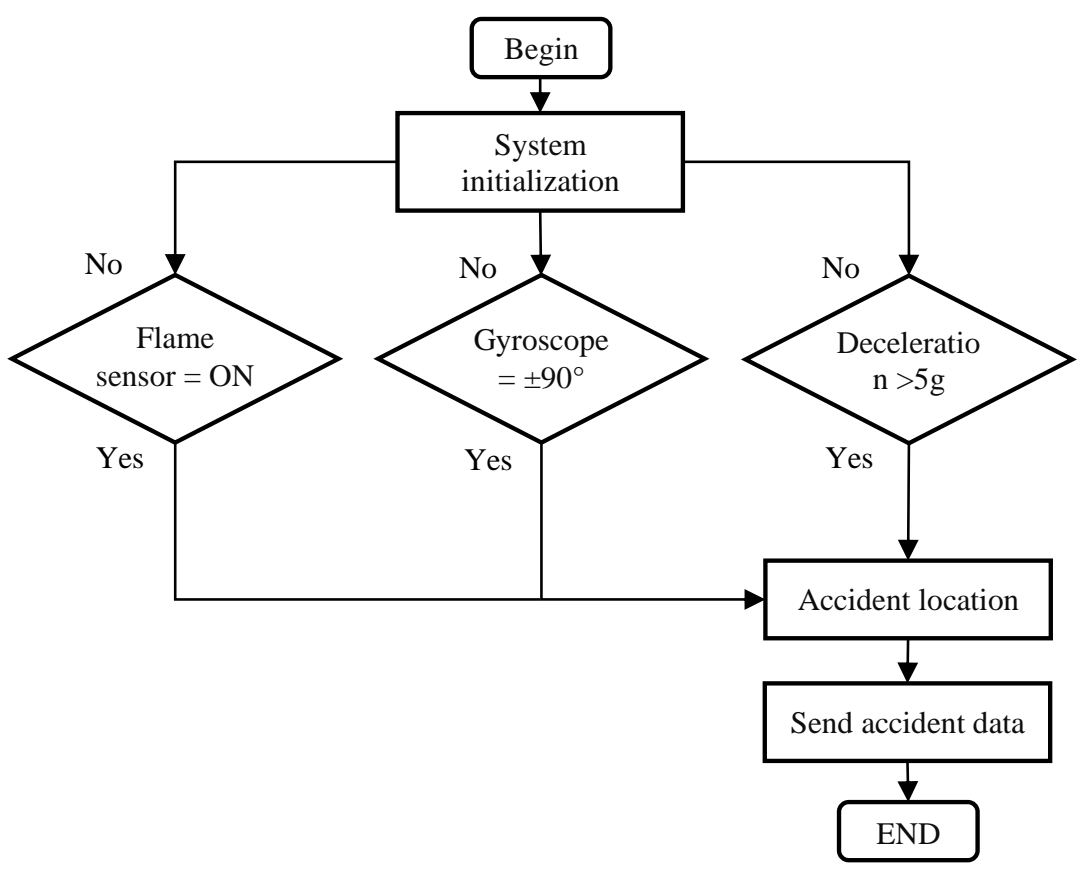

Figure 4. Diagram of the accident detection algorithm

\section{RESULTS AND DISCUSSION}

A reliable accident detection and warning system has been designed and presented in this study, the importance of such system is summarized in the use of the acceleration as a primary factor in determining the occurrence of road accidents. It also combines the main advantages of smartphones-based solution and builtin unit systems, both low-cost and a precise way to extract the real-time vehicle speed.

To determine the acceleration of the vehicle, an accident detection system was created and tested. Two variables are utilized to record the beginning and final speeds, while the initial variable takes after 140 milliseconds the value of the previous final velocity, which in turn updates its value, both speeds are necessary to calculate acceleration using an (2). The system has been assessed at different velocities by means of a medium vehicle braking, the following statistics indicate speed change and calculated acceleration following the application of the braking test at various four speeds with a Kia Carens 5-door 2015 (40kph, 60kph, 80kph and 100kph). Figures 5-8 depicted the values of the initial and the final speeds enregistred after applying a medium brake at the speeds of $40 \mathrm{kph}, 60 \mathrm{kph}, 80 \mathrm{kph}$ and $100 \mathrm{kph}$ respectively. Figures 9-12 present the result of the computed acceleration using the previous speeds values.

The minimum speed change needed to achieve the accident case which is $5 \mathrm{~g}$ in 140 milliseconds in our system must be $25 \mathrm{kph}$ at least, even the deceleration value in the test of a severe brake can't achieve this value. The highest deceleration value registered after testing the system is $-1 \mathrm{~g}$ following a medium brake at $80 \mathrm{kph}$ when the change between the initial and the final speed in this case was $5 \mathrm{kph}$. 


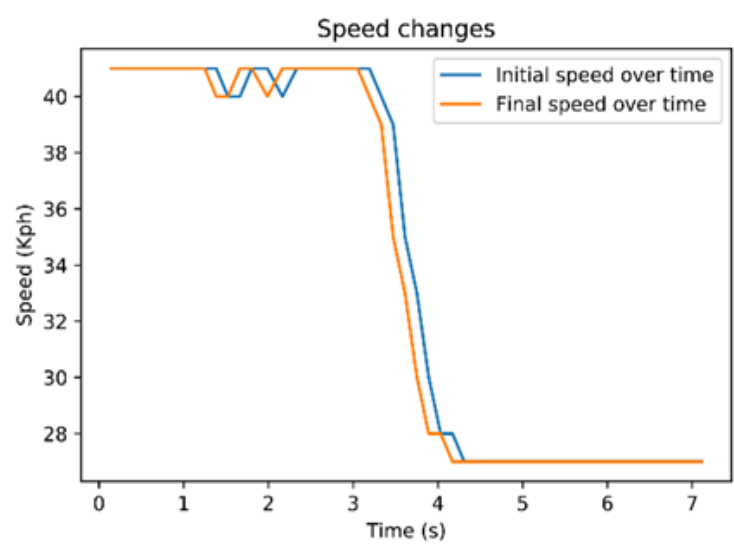

Figure 5. Speed change for the medium brake at $40 \mathrm{kph}$

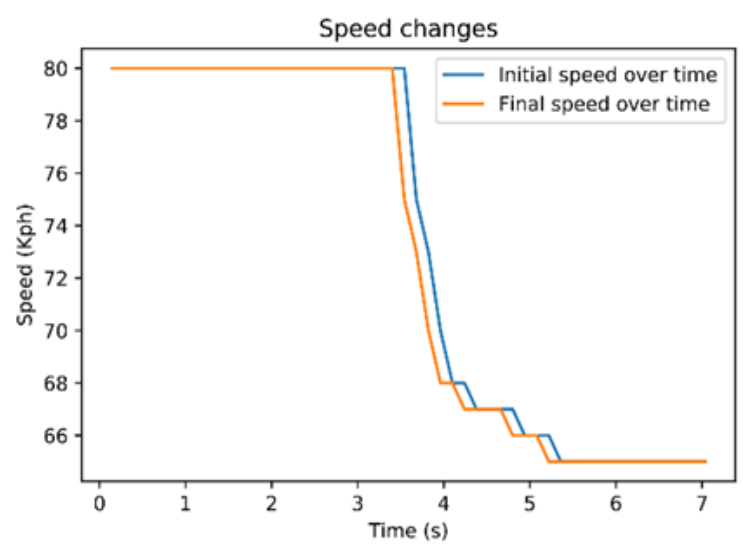

Figure 7. Speed change for the medium brake at $80 \mathrm{kph}$

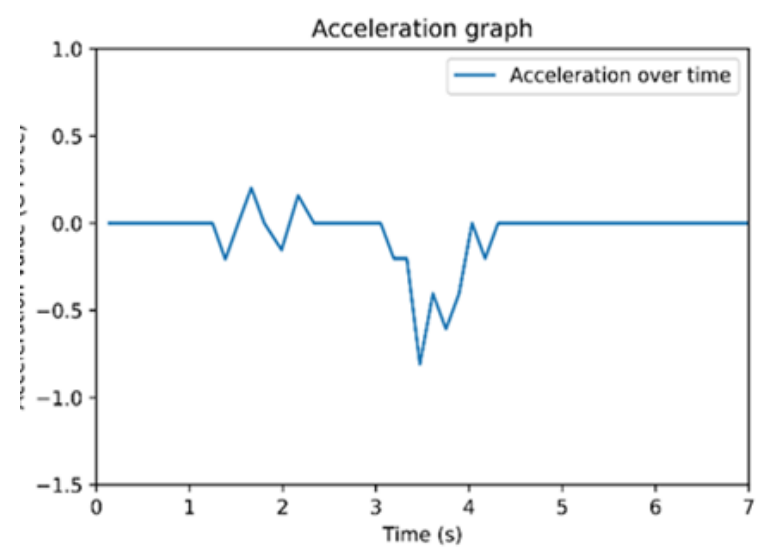

Figure 9. Acceleration for the medium brake at $40 \mathrm{kph}$

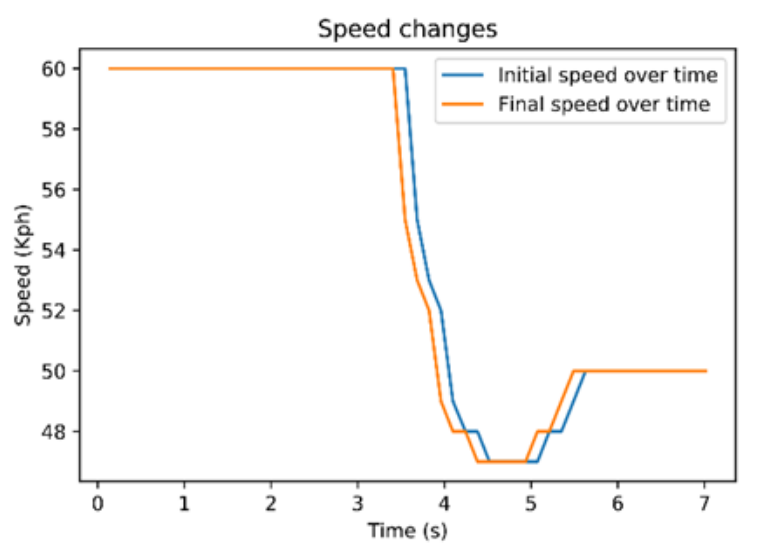

Figure 6. Speed change for the medium brake at $60 \mathrm{kph}$

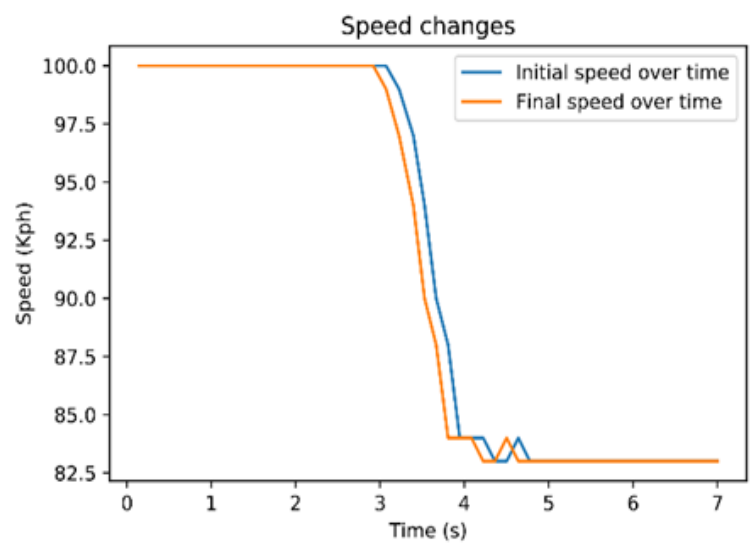

Figure 8. Speed change for the medium brake at $100 \mathrm{kph}$

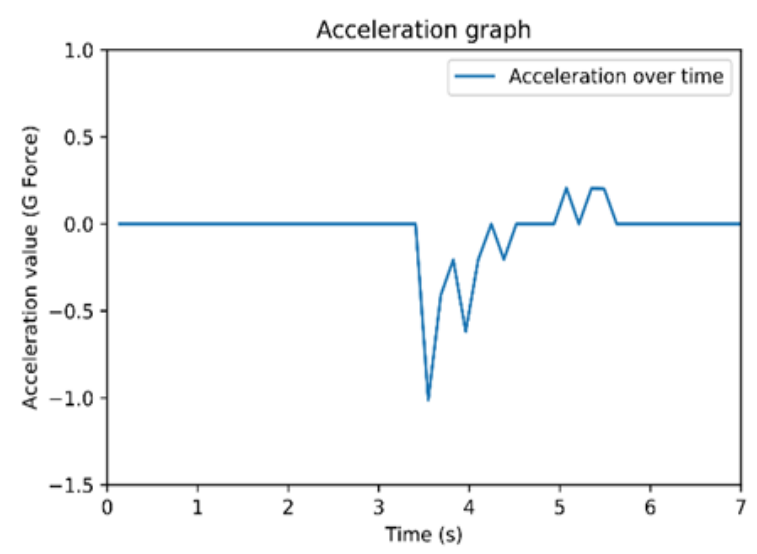

Figure 10. Acceleration for the medium brake at $60 \mathrm{kph}$ 


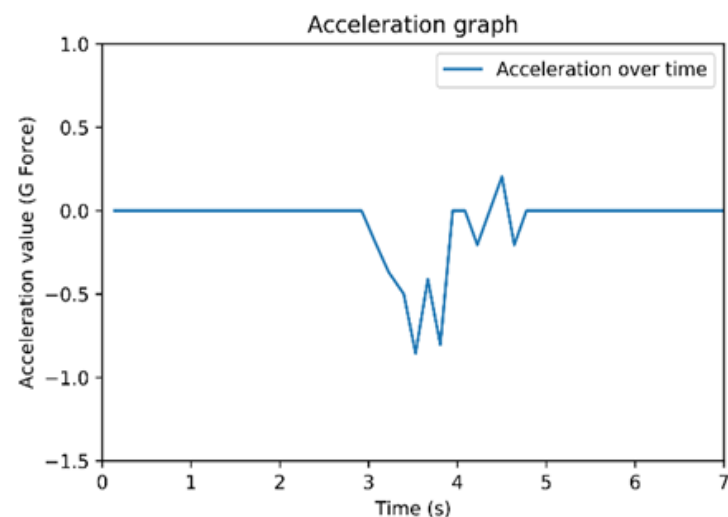

Figure 11. Acceleration for the medium brake at $80 \mathrm{kph}$

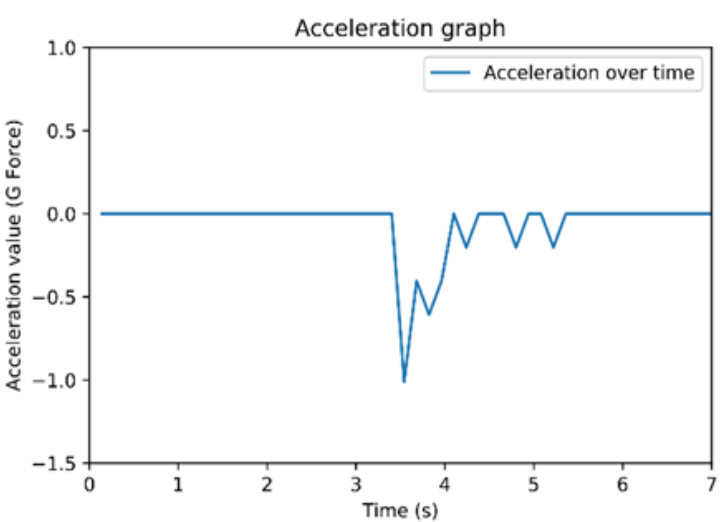

Figure 12. Acceleration for the medium brake at $100 \mathrm{kph}$

\section{CONCLUSION}

The authors developed an accident detection and location system in this article that provides a strong detection mechanism for road accidents with the use of acceleration data and rapid reporting utilizing GPS as well as GSM technologies. The key addition to our system was the notion of leveraging the actual speed data collected by means of the OBD-II system in order to compute acceleration to identify the accident. We have profited from both mobile phone and Built-In solutions advantages, mobility and robustness. And since the system uses the OBD-II standard, it can be installed in any car whatever its model. The system has been installed and tested in a real vehicle by the application of a medium brake at varied speeds due to the difficulty of its application in real accident. In order to determine the severity of accidents, the next effort rests in the integration of a machine learning model, which employs acceleration values and many other features that the system may offer to forecast the severity of the accident.

\section{ACKNOWLEDGEMENTS}

The TIT and FABLAB laboratories of Tahri Mohamed University of Bechar are ostensibly supporting this study.

\section{REFERENCES}

[1] A. Meena, S. Iyer, M. Nimje, S. Jogjekar, S. Jagtap, and M. Rahman, "Automatic Accident Detection and Reporting Framework for Two Wheelers," In: IEEE International Conference on Advanced Communication Control and Computing Technologies (ICACCCT), 2014, pp. 962-967, doi: 10.1109/ICACCCT.2014.7019237.

[2] C. R. Keerthi, G. Shanmukh, R. Sivaram, "Various Accident Detection Technologies and Recovery Systems with Victim Analysis," International Journal of Advanced Trends in Computer Science and Engineering (JJATCSE), Special Issue of ICCSIE 2013 - Held during 24 May, 2013 Bangalore, India.

[3] World Health Organization, "Glopal Status Report on Road Safety," Geneva, 2009.

[4] A. Bonyár, A. Géczy, O. Krammer, H. Sántha, B. Illés, and J Kámán, "A Review on Current eCall Systems for Autonomous Car Accident Detection," In: 40th International Spring Seminar on Electronics Technology (ISSE), pp. 1-8, 2017, doi: 10.1109/ISSE.2017.8000985.

[5] C. Thompson, J. White, B. Dougherty, A. Albright, and D. C. Schmidt, "Using Smartphones to Detect Car Accidents and Provide Situational Awareness to Emergency Responders," International Conference on Mobile Wireless Middleware, Operating Systems, and Applications, pp. 29-42, Vanderbilt University, Nashville, TN USA, 2010, doi: 10.1007/978-3-642-17758-3_3.

[6] R. K. Megalingam, R. N. Nair, and S. M. Prakhya, "Wireless Vehicular Accident Detection and Reporting System," In: 2010 International Conference on Mechanical and Electrical Technology (ICMET 2010), 2010, pp. 636-640, doi: 10.1109/ICMET.2010.5598437.

[7] D. A. Whitney and J. J Pisano TASC, Inc., Reading, Massachusetts, "Auto Alert: Automated Acoustic Detection ofIncidents," IDEA project, 1995.

[8] P. Kremonas and J. Pâris, "Everything you wanted to ask, but did not know how...," In: the European Emergency Number Association (EENA), 2015.

[9] J. White, C. Thompson, H. Turner, B. Dougherty, and D. C. Schmidt, "WreckWatch : Automatic Traffic Accident Detection and Notification with Smartphones," In: Springer Science+Business Media, LLC, pp. 285-303, 2011, doi: 10.1007/s11036-011-0304-8. 
[10] F. Aloul, I. Zualkernan, R. Abu-salma, Al-ali H, and Al-merri M, "iBump : Smartphone application to detect car accidents," In: Computer and Electrical Engineering, vol. 43, pp. 66-75, 2015, doi: 10.1016/j.compeleceng.2015.03.003.

[11] J. R. Kwapisz, G. M. Weiss, and S. A. Moore, "Activity recognition using cell phone accelerometers," $A C M$ SIGKDD Explorations Newsletter, vol. 12, no. 2, pp. 74-82, December 2011

[12] M. Fazeen, B. Gozick, R. Dantu, M. Bhukhiya, and M. C. González, "Safe driving using mobile phones," IEEE Trans. Intelligent Transportation Systems, vol. 13, no. 3, pp. 1462-1468, September 2012, doi: 10.1109/TITS.2012.2187640.

[13] B. Fernandes, M. Alam, V. Gomes, J. Ferreira, and A. Oliveira, "Automatic accident detection with multi-modal alert system implementation for ITS," In: Vehicular Communications, vol. 3, pp. 1-11, 2016, doi: 10.1016/j.vehcom.2015.11.001.

[14] S. Chaklader, J. Alam, M. Islam, and A. S. Sabbir, "Black box: an emergency rescue dispatch system for road vehicles for instant notification of road accidents and post crash analysis," in: 2014 International Conference on Informatics, Electron-ics \& Vision (ICIEV), IEEE, 2014, pp. 1-6, doi: 10.1109/TITS.2012.2187640.

[15] J. L. Mazher Iqbal and S. Heena Kousar, "Automatic Vehicle Accident Detection and Reporting With Black Box," In: International Journal of Applied Engineering Research, vol. 10, no. 87, 139-145, 2015.

[16] S. Amin and J. Jalil, "Accident Detection and Reporting System using GPS, GPRS and GSM Technology," In: IEEE/OSA/IAPR International Conference on Informatics, Electronics \& Vision, 2012, 640-643, doi: 10.1109/ICIEV.2012.6317382.

[17] B. K. Drosos and C. Malesios, "Measuring the Accuracy and Precision of the Garmin GPS positioning in Forested Areas: A Case Study in Taxiarchis - Vrastama University Forest," In: Journal of Environmental Science and Engineering, vol. 1, no. 4, 566-576, 2012.

[18] M. Syedul Amin, M. B. Ibne Reaz, S. S. Nasir and M. A. Sobhan Bhuiyan, "Low Cost GPS/IMU Integrated Accident Detection and Location System," In: Indian Journal of Science and Technology, vol. 9, no. 10, 1-9, 2016, doi: 10.17485/ijst/2016/v9i10/80221.

[19] M. S. Amin, M. A. S. Bhuiyan, M. B. I. Reaz, S. ans S. S. Nasir, "GPS and map matching based vehicle accident detection system," IEEE 2013 Student Conference on Research and Development (SCOReD), Kuala Lumpur, Malaysia. 2013 Dec 16-17, p. 520-3, doi: 10.1109/SCOReD.2013.7002645.

[20] A. EI-Rabban, "Introduction to GPS: The Global Positioning System," 2nd Ed, Artech House, 2009.

[21] H. Motte, J. Wyffels, De Strycker L, and J. Goemaere, "Evaluating GPS Data in Indoor Environments," In Advances in Electrical and Computer Engineering, vol. 11, no. 3, pp. 25-28, 2011, doi: 10.4316/aece.2011.03004.

[22] S. G. Fernandez, R. Palanisamy, and K. Vijayakumar, "GPS \& GSM Based Accident Detection And Auto Intimation," In: Indonesian Journal of Electrical Engineering and Computer Science (IJEECS), vol. 11, no. 1, 336361, 2018, doi: 10.11591/ijeecs.v11.i1.pp356-361.

[23] J. Zaldivar, C. T. Calafate, Juan Carlos Cano and Pietro Manzoni, "Providing Accident Detection in Vehicular Networks Through OBD-II Devices and Android-based Smartphones," In: 5th IEEE Workshop On User MObility and VEhicular Networks, pp. 813-819, 2011, doi: 10.1109/LCN.2011.6115556.

[24] C. Spelta, V. Manzoni, A. Corti, A. Goggi, and S. Savaresi, "Smartphone-based vehicle-to-driver/environment interaction system for motorcycles," Embedded Systems Letters, IEEE, vol. 2, no. 2, pp. 39-42, 2010, doi: 10.1109/LES.2010.2052019.

[25] P. S. Bokare and A. K. Maurya, "Acceleration-Deceleration Behaviour of Various Vehicle Types," In: Transportation Research Procedia, vol. 25, no. 1, 2017, 4733-4749, doi: 10.1016/J.TRPRO.2017.05.486.

[26] S. Samuels, and J. Jarvis, "Acceleration and deceleration of modern vehicles," Technical Report, Australian road research institute, Report 86, 1978.

[27] J. Searle, "Equations of speed, time and distance for vehicles under maximum acceleration," Advances in Safety Technology, SP-1433, pp, 1-7, 1999.

[28] W. T. Hollowell, et al., "Updated review of potential test procedures for FMVSS," In: Office of Vehicle Safety Research National Highway Traffic Safety Administration (NHTSA), no. 208, 1999.

\section{BIOGRAPHIES OF AUTHORS}

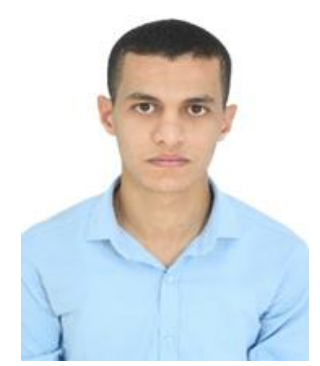

Mr. Kadri Ibrahim is a third-year doctoral student at Tahri Mohamed of Bechar, Algeria, studying Data Processing and Telecommunications. His areas of expertise include embedded systems and telecommunications. He graduated from the University of Bechar, Algeria, in 2017 with a master's degree in Digital Communication Systems. The Laboratory of Data Processing \& Telecommunications (LTIT) is located at the University of Tahri Mohamed, Bechar, Algeria. 


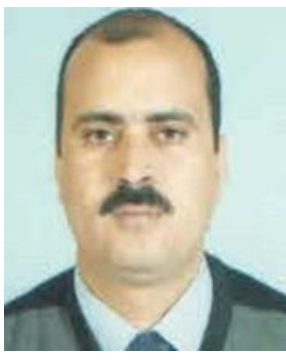

Dr. Kadri Boufeldja was born in 1972 in Bechar, Algeria. He earned a Majister degree from Tlemcen's Abou Bekrbelkaid University in 1998. (Algeria). He joined the Electronic Institute at Bechar University (Algeria) in 1999 and is currently an associate professor there. In addition to modeling and optimization of antenna arrays, heuristic methods are among his major areas of interest.

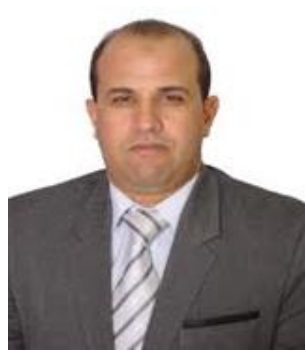

Pr. Beladgham Mohamed was born in the Algerian town of Tlemcen. He earned a diploma in electrical engineering from at Tlemcen's university, Algeria, followed by a master's degree in systems and signals and a Phd in electronics from the same university, all in 2012. He started as an Associate Professor at Algeria's Bechar University. Currently, he is a Professor of Electrical Engineering at Bechar University and conducts research in the LTIT Laboratory at Bechar University Tahri Mohammed. He is interested in video and image processing, medical pictures segmentation, biomedical imaging, biometric systems, transform wavelets and optimal encoders. Information about the contact: beladgham.tlm@gmail.com

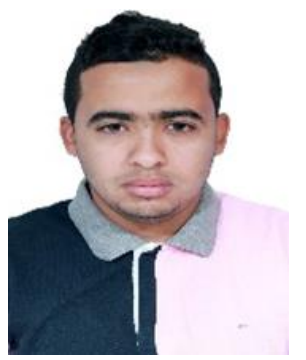

Mr. Dahmane Oussama was born in the Algerian city of Bechar. In 2017, he earned a Master's degree in numerical communication systems from Algeria's University Tahri Mouhamed Bechar. $\mathrm{He}$ is currently working on his doctorate at the University of Bechar in Algeria. Image and video processing, image segmentation, medical image compression, biomedical imaging, and deep learning are among his key interests. Email: dahmane.oussama@univ-bechar.dz Correspondence address: Bechar University, Department of Electronics, Bechar, Algeria 